\title{
Advances in novel antibiotics to treat multidrug-resistant gram-negative bacterial infections
}

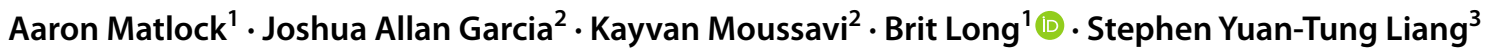

Received: 21 October 2020 / Accepted: 16 April 2021 / Published online: 6 May 2021

(c) This is a U.S. government work and not under copyright protection in the U.S.; foreign copyright protection may apply 2021

\begin{abstract}
Antimicrobial resistance is a growing threat to public health and an increasingly common problem for acute care physicians to confront. Several novel antibiotics have been approved in the past decade to combat these infections; however, physicians may be unfamiliar with how to appropriately utilize them. The purpose of this review is to evaluate novel antibiotics active against resistant gram-negative bacteria and highlight clinical information regarding their use in the acute care setting. This review focuses on novel antibiotics useful in the treatment of infections caused by resistant gram-negative organisms that may be seen in the acute care setting. These novel antibiotics include ceftolozane/tazobactam, ceftazidime/avibactam, meropenem/vaborbactam, imipenem/cilistatin/relebactam, cefiderocol, plazomicin, eravacycline, and omadacycline. Acute care physicians should be familiar with these novel antibiotics so they can utilize them appropriately.
\end{abstract}

Keywords Antibiotics $\cdot$ Resistance $\cdot$ Novel $\cdot$ Multidrug-resistant

\section{Introduction}

Antimicrobial resistance presents a critical threat to public health, and as the scope of this issue continues to grow, acute care physicians are likely to encounter resistant infections with increasing frequency. In 2019, the United States

Brit Long

brit.long@yahoo.com

Aaron Matlock

aaron.grey.matlock@gmail.com

Joshua Allan Garcia

jgarcia@ketchum.edu

Kayvan Moussavi

kmoussavi@ketchum.edu

Stephen Yuan-Tung Liang

syliang@wustl.edu

1 Department of Emergency Medicine, Brooke Army Medical Center, 3841 Roger Brooke Dr, Fort Sam Houston, TX 78234, USA

2 Assistant Professor, Department of Pharmacy Practice, Marshall B. Ketchum University College of Pharmacy, Fullerton, CA, USA

3 Department of Emergency Medicine and Division of Infectious Diseases, Department of Medicine, Washington University School of Medicine, St. Louis, MO, USA
Centers for Disease Control and Prevention (CDC) released a report titled "Antibiotic Resistance Threats in the United States," a document outlining emerging and extant trends in antimicrobial resistance [1]. Several findings suggest progress, such as a decrease in deaths caused by antibioticresistant infections since the prior report in 2013, but the data are overall sobering [1]. Annually, 2.8 million infections and 35,000 deaths in the United States are attributable to antibiotic-resistant organisms, and the incidence of multidrug-resistant (MDR) infections has increased considerably [1]. For instance, infections caused by extended spectrum beta lactamase (ESBL) producing Escherichia coli increased by $50 \%$ since the 2013 report [1].

Antimicrobial resistance is a natural phenomenon and spans a broad array of human pathogens. While resistant strains of fungi (e.g. Candida auris), gram-positive bacteria (e.g. Staphylococcus aureus), and anaerobic species (e.g. Clostridioides difficile) all represent important threats to public health, infections caused by antibiotic-resistant gram-negative aerobes have proven particularly vexing to the treating clinician [2]. Pseudomonas aeruginosa and Enterobacterales species such as Klebsiella pneumoniae and $E$. coli demonstrate diverse mechanisms of antibiotic resistance, both innate and acquired [2]. Many resistance factors are readily transmitted to other related organisms, and additive or synergistic resistance to multiple classes of 
antimicrobials can result, even to drugs typically reserved as a last line of defense against infection [3-7]. Within this landscape, it is critical that acute care physicians be aware of changing trends in antimicrobial resistance and options for treatment of resistant organisms. Here, we describe key trends in gram-negative bacterial resistance and review a selection of antimicrobial drugs released in the past decade that may be of utility in the treatment of emergency department and hospitalized patients with multidrug-resistant infections. We focus on these drugs as they pertain to infectious disease syndromes commonly encountered in the acute care setting, such as pneumonia, intraabdominal infections, and infections of the urinary tract and skin structures.

\section{Methods}

A literature review of the PubMed and Google Scholar databases was performed with search date from September 1, 2010 to September 1, 2020 for articles using the keywords 'novel antibiotic' OR 'ceftolozane/tazobactam' OR 'ceftazidime/avibactam' OR 'meropenem/vaborbactam' OR 'imipenem/cilistatin/relebactam' OR 'cefiderocol' OR 'plazomicin' OR 'eravacycline' OR 'omadacycline' OR 'antibiotic resistance' for production of this narrative review. We did not include articles related to antimicrobial drugs whose spectra of activity is primarily against gram-positive pathogens or whose antimicrobial activity does not include multidrug-resistant pathogens. Authors included case reports and series, retrospective and prospective studies, systematic reviews and meta-analyses, clinical guidelines, and other narrative reviews. The literature search was restricted to studies published in English. Initial literature search revealed over 6197 articles. Authors reviewed all relevant articles and decided which studies to include for the review by consensus, with focus on articles relevant to the acute care setting, including guidelines. A total of 67 resources were selected for inclusion in this review. We specified drug-resistant pathogens according to standardized definitions as follows $[8,9]$ :

Multidrug-resistant (MDR)-Resistant to an agent in $\geq 3$ classes of antibiotics

Extensively drug resistant (XDR)-Resistant to an agent in all but 1-2 classes of antibiotics

Pan-drug resistant (PDR)-Resistant to all classes of antibiotics

\section{Discussion}

\section{MDR bacteria in the acute care setting}

While bacterial resistance threatens to degrade the effectiveness of antibiotics against a growing number of human pathogens, several gram-negative MDR pathogens are of particular concern to the acute care physician, given their increasing prevalence, virulence, and the limited available drugs to treat them $[2,10-12]$. These include extendedspectrum- $\beta$-lactamase producing Enterobacterales (ESBLE), carbapenem-resistant Enterobacterales (CRE), and $P$. aeruginosa with difficult-to-treat resistance (DTR-P. aeruginosa) [2, 10-12]. ESBLs are enzymes that inactivate most beta-lactam agents, including penicillins, cephalosporins, and aztreonam, but not non- $\beta$-lactam agents, such as fluroquinolones, trimethoprim-sulfamethoxazole, nitrofurantoin, or aminoglycosides $[10,12]$. $\beta$-lactamases are commonly classified according to the Ambler molecular classification scheme or Bush-Jacoby-Medieros functional classification system [11, 12]. The Ambler classification is based on amino-acid sequence similarities while the Bush classification is based on functionality [12]. Ambler classification is divided into four molecular classes (A-D), with class $\mathrm{A}, \mathrm{C}$, and D utilizing a serine moiety and B consisting of a metalloenzymatic zinc ion at its active site [12, 13]. Bush classification is divided into three major groups: Group 1 cephalosporinases (Ambler class C), Group 2 serine $\beta$-lactamases (Ambler classes A and D), and Group 3 metallo- $\beta$-lactamases (Amber class B), with each group containing further subdivisions. Examples of ESBLs include CTX-M (Ambler class A), TEM (Ambler class A), SHV (Ambler class A), and OXA (Ambler class D) [5, 10, 12, 13].

CRE denotes members of the Enterobacterales order resistant to at least one carbapenem antibiotic or producing a carbapenemase enzyme. CRE may be resistant to some carbapenems but not others (e.g. resistant to ertapenem but sensitive to meropenem). This group of pathogens can be divided into organisms that are carbapenemase-producing and those that are not carbapenemase-producing [10]. Notable carbapenemases include Klebsiella pneumoniae carbapenemases (KPCs) (Ambler class A), New Delhi metallo- $\beta$-lactamases (NDMs) (Ambler class B), Verona integrin-encoded metallo- $\beta$-lactamases (VIMs) (Ambler class B), imipenem-hydrolyzing metallo- $\beta$-lactamases (IMPs) (Ambler class B), and oxacillinase (OXA-48-like) carbapenemases (Ambler class D) [10, 13]. Identifying if a CRE isolate is carbapenemase-producing and which carbapenemase is produced can be important when determining optimal treatment [10].

DTR- $P$. aeruginosa is defined as non-susceptibility to piperacillin-tazobactam, ceftazidime, cefepime, 
aztreonam, meropenem, imipenem-cilastatin, ciprofloxacin, and levofloxacin [10]. Resistance can be due to multiple mechanisms including $\beta$-lactamase production, antibiotic efflux, porin alterations, and target site modification [2]. AmpC $\beta$-lactamase (Ambler class $\mathrm{C}$ ) provides resistance to penicillins and cephalosporins, including antipseudomonal agents such as piperacillin-tazobactam and ceftazidime [2,13]. Resistance to carbapenems can result from downregulation of porins like $\mathrm{OprD}$, upregulation of efflux pumps like those that belong to the RND-type efflux system (e.g. MexAB-OprM), or a combination of both mechanisms [2]. P. aeruginosa can also alter antibiotic target sites on ribosomal subunits or topoisomerases to prevent binding of aminoglycosides and fluoroquinolones, respectively. These combinations of resistance mechanisms highlight why DTR-P. aeruginosa can have so few treatment options $[2,10]$.

The myriad mechanisms of gram-negative bacterial resistance underscore the unique challenges facing the acute care physician in the treatment of MDR infections, as well as the ongoing threat to public health. Accordingly, the CDC tracks and reports data related to these pathogens, among other drug-resistant bacteria. Some of the CDC's reporting on the public health impact of these pathogens is summarized in Table $1[1,2,4,5]$. A number of patient factors have been identified that increase the risk for infections caused by MDR pathogens, such as recent institutional care and/or exposure to antibiotics, history of MDR infections, and immunocompromised states, such as diabetes mellitus [7, 8, 14-19]. Risk factors may differ somewhat by pathogen and disease state; for instance, chronic lung disease (e.g. chronic obstructive pulmonary disease, bronchiectasis, cystic fibrosis) is a noted risk factor for community-acquired pneumonia caused by MDR pathogens, while recent history of an outpatient genitourinary or gastrointestinal procedure has been cited as a risk factor for urinary tract infections caused by resistant bacteria $[14,18]$. Assessment for such risk factors and awareness of local resistance patterns is critical, as antimicrobial therapy may differ according to risk profile.

While many MDR infections are associated with hospitalization and long-term residential care, they are certainly not exclusive to these settings. Community-acquired spread of MDR gram-negative infections is now common, and such cases can occur in patients lacking the traditional risk factors associated with MDR organisms [14, 20-23]. In the acute care setting, these infections frequently manifest in the urinary tract $[14,20-23]$. In two recent EDbased series, $35-44 \%$ of urinary ESBL-producing E. coli isolates originated from patients without traditional risk factors for MDR infections [21, 22]. Not surprisingly, discordant initial antibiotic therapy was commonly reported in these cohorts [21,22].

In an effort to identify patients at higher risk for infection caused by drug-resistant bacteria, a number of disease and drug-specific scoring systems have been derived. While many of these scores lack external validation, review of the associated patient risk factors for each can provide a conceptual framework for assessing an individual patient's risk of resistant pathogens. For instance, scores derived to predict fluoroquinolone (FQ) resistance and ESBL-producing Enterobacterales in bloodstream infections identified fluoroquinolone use in the preceding 90 days as a risk factor for both conditions $[16,17]$. Similarly, a score derived to predict FQ resistance in urinary tract infections (UTI) identified prior FQ use as a strong predictor of FQ-resistant UTIs [18].

Table 1 Examples of multi-drug resistant organisms

ESBL-producing Enterobacterales

MDR P. aeruginosa

Colistin-resistant E. coli

Carbapenem-resistant Acinetobacter baumannii
Resistant to most beta-lactam antibiotics, including penicillins, cephalosporins, and aztreonam. ESBLs are commonly associated with additional resistance to other drug classes as well, including fluoroquinolones. Hundreds of ESBLs have been identified, and they now comprise:

$13.4 \%$ of hospital-acquired $E$. coli infections

$20 \%$ of hospital-acquired $K$. pneumoniae infections

Resistant to carbapenem antibiotics among others, including those inhibited by ESBL. CREs comprise:

$8.7 \%$ of hospital-acquired K. pneumoniae infections

$0.7 \%$ of hospital-acquired E. coli infections

$P$. aeruginosa is intrinsically resistant to many antibiotics, and additional resistance factors have compounded the problem

$19.3 \%$ of hospital-acquired isolates are resistant to carbapenems

Although rare, plasmid-borne colistin-resistance has been documented in E. coli Colistin is often a drug of last resort for MDR gram-negative infections

A. baumannii is intrinsically resistant to many antibiotics, and carbapenem resistance can spread among bacteria via mobile resistance elements

Limited treatment options exist for carbapenem-resistant strains

8500 U.S. cases of A. baumannii were identified in 2019 alone 
A number of criteria and scoring systems have similarly been developed in patients with pneumonia, but some of these scoring systems have inadequate sensitivity and/ or specificity to be clinically useful. However, the Drug Resistance in Pneumonia (DRIP) [Table 2] score has demonstrated comparatively promising test characteristics and clinicians can consider its use to augment clinical judgement. In the derivation and validation study of this decision instrument, a DRIP score of 4 or greater has been shown to identify patients at risk for pneumonia due to a drug-resistant pathogen with a sensitivity and specificity of $82 \%$ and $81 \%$, respectively. However, it should be noted that this risk assessment tool is not specific to gram-negative bacteria, as these pathogens accounted for only $23 \%$ and $34 \%$ of positive cultures in the derivation and validation set, respectively $[15,24]$.

\section{Treating MDR infections}

Infections caused by MDR organisms are associated with increased mortality, hospital length of stay, and health care costs [8, 25-27]. Administration of appropriate antibiotics prior to intensive care unit (ICU) admission has been associated with decreased mortality in severe sepsis and septic shock, a finding that underscores the importance of considering appropriate antibiotic spectra in patients at risk for MDR infections [28]. Various rapid detection methods, such as polymerase chain reaction assays and mass spectrometry, have demonstrated efficacy in identifying pathogens earlier than traditional culture methods, and these technologies may be of future use in guiding ED antibiotic choices [7].

The U.S. Food and Drug Administration (FDA) has approved several novel antibiotics in the past decade with activity against MDR gram-negative bacteria. Many of these drugs are currently reserved for exceptional use (e.g., in patients with documented MDR or extensively drug-resistant

Table 2 DRIP score

\begin{tabular}{ll}
\hline Characteristic & Points \\
\hline Antibiotic use within previous 60 days & 2 \\
Residence in a long-term care facility & 2 \\
Tube feeding & 2 \\
Prior infection with drug-resistant pathogen within previous & 2 \\
$\quad$ year & 1 \\
Hospitalization within previous 60 days & 1 \\
Chronic pulmonary disease & 1 \\
Poor functional status & 1 \\
Gastric acid suppression & 1 \\
Wound care & 1 \\
MRSA colonization within previous year & \\
\hline
\end{tabular}

Adapted from Webb et al. 2016 [2]
(XDR) infection), and coordination with an infectious disease specialist and/or clinical pharmacist is advisable when considering their use. Accordingly, we do not recommend empiric use of these drugs outside of these scenarios. However, as resistance patterns evolve, some of these agents could potentially be used to supplement or replace existing empiric antibiotic regimens in the hospital, either on a caseby-case basis, or as part of more broadly applied protocols. Thus, the following primer is intended to familiarize acute care physicians with some of the newer antibiotics being used in the treatment of select patients with MDR infections.

\section{Novel antibiotics}

This drug-focused review highlights agents targeting resistant gram-negative organisms, such as MDR P. aeruginosa or carbapenem-resistant Enterobacterales, and disease states commonly treated in the hospital (e.g. urinary tract infections, community acquired bacterial pneumonia). This guide is not a comprehensive list of all drug information, but rather is intended to serve as a practical reference for the practicing clinician. Tables 3 and 4 provide a summary of these medications.

\section{Ceftolozane/tazobactam}

Ceftolozane/tazobactam (Zerbaxa ${ }^{\mathrm{TM}}$ ) is a novel cephalosporin plus beta-lactamase inhibitor combination approved in 2014 [29]. Ceftolazone/tazobactam was first approved for complicated urinary tract infections (cUTI)/pyelonephritis, complicated intra-abdominal infections (cIAI), and later hospital acquired bacterial pneumonia (HABP)/ventilatorassociated bacterial pneumonia (VABP) [29]. Ceftolozane/ tazobactam was tested against levofloxacin for cUTI/pyelonephritis and against meropenem for pneumonia and cIAI (in combination with metronidazole) for the aforementioned indications. All patients in the trials were treated in acute care settings without mention of treatment in the emergency department. Ceftolozane is a novel cephalosporin antibiotic that exhibits its antibiotic effect in a similar method to other beta-lactams: inhibition of transpeptidase (a penicillin binding protein, or PBP) leading to the inhibition of bacterial cell wall and eventual bacterial death [30-32]. Ceftolozane's unique chemical structure allows for greater binding to PBPs as compared to other beta-lactams such as ceftazidime and imipenem [33]. When combined with the beta-lactamase inhibitor tazobactam, its spectrum of activity is increased, covering a wide variety of gram-positive and gram-negative organisms, such as Streptococcus spp., methicillin-sensitive S. aureus (MSSA), E. coli, Klebsiella spp., and Haemophilus influenzae [29]. However, ceftolazone/tazobactam is more notable for its activity against ESBL Enterobacterales and MDR P. aeruginosa [34]. Unfortunately, this drug does 
Table 3 Highlights of Novel Antibiotics

\begin{tabular}{|c|c|c|c|c|}
\hline $\begin{array}{l}\text { Generic drug name (brand } \\
\text { name) }\end{array}$ & Class/mechanism of action & Notable gram $(+)$ coverage & Notable gram (-) coverage & $\begin{array}{l}\text { Approved } \\
\text { infection site/ } \\
\text { syndrome }\end{array}$ \\
\hline $\begin{array}{l}\text { Ceftolozane/tazobactam } \\
\left(\text { Zerbaxa }^{\mathrm{TM}}\right)\end{array}$ & $\begin{array}{l}\text { Class: Novel cephalo- } \\
\text { sporin + beta-lactamase } \\
\text { inhibitor } \\
\text { MOA: Inhibition of cell wall } \\
\text { production }\end{array}$ & $\begin{array}{l}\text {-MSSA } \\
\text {-Streptococcus spp. }\end{array}$ & $\begin{array}{l}\text {-ESBL } \\
\text {-MDR P. aeruginosa }\end{array}$ & $\begin{array}{l}\text {-cUTI } \\
\text {-cIAI } \\
\text {-HABP/VABP }\end{array}$ \\
\hline $\begin{array}{l}\text { Ceftazidime/avibactam (Avy- } \\
\text { caz }^{\mathrm{TM}} \text { ) }\end{array}$ & $\begin{array}{l}\text { Class: Cephalosporin + novel } \\
\text { beta-lactamase inhibitor } \\
\text { MOA: Inhibition of cell wall } \\
\text { production }\end{array}$ & $\begin{array}{l}\text {-MSSA } \\
\text {-Streptococcus spp. }\end{array}$ & $\begin{array}{l}\text {-ESBL } \\
\text {-KPC } \\
\text {-OXA-48 carbapenemase } \\
\text {-MDR P. aeruginosa (less } \\
\text { than ceftolozane/avibactam) } \\
\text {-MDR Acinetobacter spp. }\end{array}$ & $\begin{array}{l}\text {-cUTI } \\
\text {-cIAI } \\
\text {-HABP/VABP }\end{array}$ \\
\hline $\begin{array}{l}\text { Meropenem/ } \\
\text { vaborbactam } \\
\left(\text { Vabomere }^{\mathrm{TM}}\right)\end{array}$ & $\begin{array}{l}\text { Class: Carbapenem }+ \text { novel } \\
\text { beta-lactamase inhibitor } \\
\text { MOA: Inhibition of cell wall } \\
\text { production }\end{array}$ & $\begin{array}{l}\text {-MSSA } \\
\text {-Streptococcus spp. } \\
\text {-Enterococcus spp. (non- } \\
\text { VRE) }\end{array}$ & $\begin{array}{l}\text {-ESBL } \\
\text {-KPC } \\
\text {-P. aeruginosa (non-MDR) } \\
\text {-Acinetobacter spp. (non- } \\
\text { MDR) }\end{array}$ & -cUTI \\
\hline $\begin{array}{l}\text { Imipenem/cilistatin/ } \\
\text { relebactam }\left(\text { Recarbrio }^{\mathrm{TM}}\right)\end{array}$ & $\begin{array}{l}\text { Class: Carbapenem }+ \text { novel } \\
\text { beta-lactamase inhibitor } \\
\text { MOA: Inhibition of cell wall } \\
\text { production }\end{array}$ & $\begin{array}{l}\text {-MSSA } \\
\text {-Streptococcus spp. }\end{array}$ & $\begin{array}{l}\text {-ESBL } \\
\text {-KPC } \\
\text {-MDR P. aeruginosa }\end{array}$ & $\begin{array}{l}\text {-cUTI } \\
\text {-cIAI } \\
\text {-HABP/VABP }\end{array}$ \\
\hline $\begin{array}{l}\text { Cefiderocol } \\
\left(\text { Fetroja }^{\circledR}\right)\end{array}$ & $\begin{array}{l}\text { Class: Novel siderophore } \\
\text { cephalosporin } \\
\text { MOA: Inhibition of cell wall } \\
\text { production via iron-binding } \\
\text { and active cell uptake }\end{array}$ & -Streptococcus spp. (in vitro) & $\begin{array}{l}\text {-P. aeruginosa } \\
\text {-A. baumannii } \\
\text {-S. maltophilia } \\
\text {-ESBL } \\
\text {-CRE (all Ambler classes) }\end{array}$ & -cUTI \\
\hline $\begin{array}{l}\text { Plazomicin } \\
\left(\text { Zemdri }^{\mathrm{TM}}\right)\end{array}$ & $\begin{array}{l}\text { Class: Aminoglycoside } \\
\text { MOA: Inhibition of } 30 \mathrm{~s} \\
\text { ribosomal subunit halting } \\
\text { protein production }\end{array}$ & -None & $\begin{array}{l}\text {-ESBL } \\
\text {-KPC } \\
\text {-OXA-48 carbapenemase } \\
\text {-Variable } P \text {. aeruginosa } \\
\quad \text { activity }\end{array}$ & -cUTI \\
\hline $\begin{array}{l}\text { Eravacycline } \\
\left.\text { (Xerava }^{\mathrm{TM}}\right)\end{array}$ & $\begin{array}{l}\text { Class: Tetracycline } \\
\text { MOA: Inhibition of } 30 \mathrm{~s} \\
\text { ribosomal subunit halting } \\
\text { protein production }\end{array}$ & $\begin{array}{l}\text {-Streptococcus spp. } \\
\text {-MRSA } \\
\text {-VRE }\end{array}$ & $\begin{array}{l}\text {-CRE (unspecified) } \\
\text {-MDR A. baumannii }\end{array}$ & -cIAI \\
\hline $\begin{array}{l}\text { Omadacycline } \\
\left(\text { Nuzyra }^{\mathrm{TM}}\right)\end{array}$ & $\begin{array}{l}\text { Class: Tetracycline } \\
\text { MOA: Inhibition of } 30 \mathrm{~s} \\
\text { ribosomal subunit halting } \\
\text { protein production }\end{array}$ & $\begin{array}{l}\text {-MSSA } \\
\text {-MRSA } \\
\text {-Streptococcus spp. }\end{array}$ & $\begin{array}{l}\text {-ESBL } \\
\text {-Acinetobacter spp. } \\
\text {-B. fragilis }\end{array}$ & $\begin{array}{l}-\mathrm{ABSSSI} \\
\text {-CABP }\end{array}$ \\
\hline
\end{tabular}

not have reliable activity for carbapenemases, MDR Acinetobacter spp., or AmpC beta-lactamases (e.g., CREs or ceftazidime-resistant Enterobacter) [35].

The side effect profile of ceftolozane/tazobactam does not differ greatly from other beta-lactam antibiotics and has generally been associated with relatively mild nausea, vomiting, and diarrhea [29]. The drug also carries warnings for hypersensitivity reactions, Clostridium difficile-associated diarrhea (CDAD), and seizures [29].

Due to its spectrum of activity, ceftolozane/tazobactam should generally be reserved for MDR $P$. aeruginosa infections rather than CRE infections. Although only indicated for cUTI, cIAI, and HABP/VABP, there have been instances of successful off-label use in bacteremia, bone/joint, and wound infections [36]. Additionally, clinicians may consider using this drug as a carbapenem-sparing treatment option if the patient has a confirmed or highly likely ESBL infection, but consideration should be given to cost and need for the future antibiotic armamentarium.

\section{Ceftazidime/avibactam}

Ceftazidime/avibactam (Avycaz ${ }^{\mathrm{TM}}$ ) is a cephalosporin + novel beta-lactamase inhibitor combination that was approved in 2015 [37]. Ceftazidime/avibactam is currently indicated for cUTI, cIAI, and most recently HABP/ VABP [37]. Ceftazidime/avibactam was tested against doripenem for $\mathrm{cUTI} /$ pyelonephritis and against meropenem 
Table 4 Novel antibiotics by approved infection site/ syndrome

\begin{tabular}{ll}
\hline Infection site/syndrome & Approved drug(s) \\
\hline Complicated urinary tract infection & Ceftolozane/taxobactam \\
& Ceftazidime/avibactam \\
& Meropenem/vaborbactam \\
& Imipenem/cilistatin/relebactam \\
Hospital/ventilator acquired bacterial pneumonia & Cefiderocol \\
& Plazomicin \\
Community-acquired bacterial pneumonia & Ceftolozane/taxobactam \\
Complicated intraabdominal infection & Ceftazidime/avibactam \\
& Imipenem/cilistatin/relebactam \\
Acute bacterial skin and skin structure infection & Omadacycline \\
\hline
\end{tabular}

for pneumonia and cIAI (in combination with metronidazole) for the aforementioned indications. All patients in the trials were treated in acute care settings without mention of treatment in the emergency department. Ceftazidime is a 3rd generation cephalosporin with activity against gramnegative bacilli, including $P$. aeruginosa [37-40]. Like other beta-lactam antibiotics, ceftazidime exerts its antibacterial effect by binding to transpeptidase (a PBP), a critical enzyme in bacterial cell wall synthesis [37]. Ceftazidime can be inactivated by a number of different beta-lactamases including AmpC, ESBLs, and all carbapenemases [37]. However, when used in combination with the novel beta-lactamase inhibitor, avibactam, the drug regains activity against a number of beta-lactamases such as ESBL and Klebsiella pneumoniae carbapenemases (KPC), as well as OXA-48 [41]. Unlike more traditional beta-lactamase inhibitors (sulbactam, tazobactam, or clavulanic acid), avibactam binds reversibly to beta-lactamases and employs a reactive urea to exert its protective mechanisms, therefore expanding the coverage of ceftazidime to a greater extent [37]. However, ceftazidime/avibactam does not have activity against Ambler Class B metallo-beta-lactamases and therefore will not cover all carbapenemases broadly [41].

From an adverse effect profile, ceftazidime/avibactam does not differ greatly from other beta-lactam antibiotics and has generally been associated with relatively mild nausea, vomiting, and diarrhea [37]. Additionally, the drug carries warnings for hypersensitivity reactions, CDAD, and seizures [37].

The spectrum of activity of ceftazidime/avibactam makes it an excellent agent for ESBLs, as well as KPC or OXA-48 carbapenemase producing Gram-negative organisms. Similar to many novel antimicrobial agents, ceftazidime/avibactam lacks activity against metallo-beta-lactamases [37]. It should also be noted that this drug appears to have worse in vitro activity against MDR $P$. aeruginosa compared to ceftolozane/tazobactam and therefore may not be the preferred agent when treating this particular organism [42, 43].

\section{Meropenem/vaborbactam}

Meropenem/vaborbactam (Vabomere ${ }^{\mathrm{TM}}$ ) is a combination antibiotic incorporating both a broad spectrum carbapenem and a novel beta-lactamase inhibitor [44]. Meropenem/ vaborbactam was approved for treatment of cUTI in 2017 by proving non-inferiority to piperacillin/tazobactam [44, 45]. Meropenem is a well-known carbapenem antibiotic with a broad spectrum of activity and stand-alone stability against a number of beta-lactamases, including ESBL [44]. Vaborbactam is a novel beta-lactamase inhibitor that utilizes boronic acid to enhance the stability of meropenem against carbapenemases such as KPC [44]. Therefore, in addition to meropenem's original activity against Streptococcus spp., MSSA, Bacteroides fragilis, Enterobacterales (including ESBLs), P. aeruginosa, and Acinetobacter spp., meropenem/vaborbactam has demonstrated sufficient activity against multiple KPCs [44].

Meropenem/vaborbactam does not have reliable activity against metallo-beta-lactamases or OXA type carbapenemases [44]. In addition, vaborbactam does not have enhanced coverage of resistant $P$. aeruginosa and Acinetobacter spp. due to combination resistance mechanisms [46]. This means that if the isolate demonstrates inherent resistance to meropenem, then it is unlikely that meropenem/ vaborbactam will have any clinical viable activity, and other agents should be considered [46].

The side effect profile of this drug does not differ greatly from other beta-lactam antibiotics and has generally been associated with relatively mild nausea, vomiting, and diarrhea [44]. It also carries warnings for hypersensitivity reactions, CDAD, and seizures (especially if the patient is managed with valproic acid or divalproex) [44]. 
Meropenem/vaborbactam is approved for cUTI and therefore can be used in both cystitis and pyelonephritis [47]. In addition, it has been shown to be an effective option in treating resistant gram-negative infections in HABP/VABP, cIAI, cUTI, and bacteremia cases compared to best available therapy (often combination or monotherapies including polymyxins, carbapenems, aminoglycosides, tigecycline, and ceftazidime/avibactam) for patients with known CRE infections. [48] Therefore, although not formally approved for these indications, clinicians may consider the use of meropenem/vaborbactam for resistant Enterobacterales (especially $\mathrm{KPC}$ ) in HABP/VABP, cIAI, and bacteremia.

\section{Plazomicin}

Plazomicin (Zemdri $\left.{ }^{\mathrm{TM}}\right)$ is a novel aminoglycoside antibiotic approved in 2016 [49]. Plazomicin exerts its antibacterial effect by inhibition of the $30 \mathrm{~s}$ ribosomal subunit, which in turn inhibits bacterial protein synthesis, much like other aminoglycosides [49]. However, plazomicin's unique chemical structure prevents inactivation by aminoglycoside modifying bacterial enzymes and enhanced spectrum of activity [49].

Like other aminoglycosides, plazomicin covers exclusively gram-negative organisms such as $E$. coli and $K$. pneumoniae [49]. Notably, it has activity against ESBLproducing organisms as well as some carbapenemases such as KPCs and OXA-48 but lacks activity against Acinetobacter spp., Stenotrophomonas spp., metallo-beta-lactamase producing organisms, and variable activity against $P$. aeruginosa [50].

Plazomicin carries the traditional boxed warnings of the aminoglycoside class: nephrotoxicity, ototoxicity, neuromuscular blockade, and fetal harm [49]. So far, no other significantly unique adverse reactions have been associated with its use [49].

Plazomicin is approved for use in cUTI in inpatients and was found to be non-inferior to meropenem as an active comparator [51]. The developers of the drug pursued approval for treatment of bacteremia but were denied this indication by the FDA Antimicrobial Drugs Advisory Committee largely due to difficulty enrolling patients and subsequent protocol changes to improve enrollment [52]. Therefore, although this drug has shown promising early results against resistant bacteria, the ruling from the FDA likely limits its current use to the treatment of cUTI.

\section{Eravacycline}

Eravacycline (Xerava ${ }^{\mathrm{TM}}$ ) is a novel tetracycline antibiotic approved in 2018 [53]. Like other tetracycline antibiotics, eravacycline exerts its antibacterial effect by inhibiting the $30 \mathrm{~s}$ ribosomal subunit for bacterial protein synthesis [53]. In terms of chemical structure, eravacycline most closely resembles tigecycline, giving it a similar spectrum of activity [53].

Like tigecycline, eravacycline adequately covers a broad array of both gram-positive and gram-negative organisms, including vancomycin-resistant Enterococci (VRE), MRSA, B. fragilis, and multidrug-resistant Enterobacterales and $A$. baumannii but lacks coverage against $P$. aeruginosa and Proteus spp [53]. Since eravacycline is not directly affected by beta-lactamases, there is evidence to suggest its effective use for carbapenemase-producing organisms, including KPC, OXA-48 and metallo-beta-lactamases, but more clinical data are needed [54].

Eravacycline has been associated with the tetracycline class warnings of tooth discoloration and inhibition of bone growth in children $<8$ years of age and CDAD [53]. The most common adverse reactions associated with its use have been infusion site reactions, nausea, and vomiting, but these have notably been observed at lower rates compared to tigecycline [53]. In addition, eravacycline has not been associated with an increased risk of mortality like tigecycline.

Eravacycline is approved for use in cIAI through demonstration of non-inferiority of clinical response in two separate clinical trials compared to ertapenem and meropenem $[55,56]$. Eravacycline may serve as an alternative to tigecycline for MDR Acinetobacter and CRE infections to avoid the increased mortality risk or if the patient is unable to tolerate tigecycline therapy. It is not indicated for the treatment of cUTI [39].

\section{Omadacycline}

Omadacycline (Nuzyra ${ }^{\mathrm{TM}}$ ) is a novel tetracycline antibiotic approved in 2018 [57]. Like other tetracycline antibiotics, omadacycline exerts its antibacterial effect by inhibiting the $30 \mathrm{~s}$ ribosomal subunit for bacterial protein synthesis, but its chemical structure allows for sustained protein synthesis inhibition even in the presence of common tetracycline efflux pumps [57].

Compared to eravacycline, omadacycline's spectrum of activity more closely resembles that of traditional tetracycline-class drugs, with some significant enhancements [57]. Omadacycline broadly covers Streptococcus spp., $S$. aureus (including MRSA), Enterobacterales, H. influenzae, $M$. catarrahlis, B. fragilis, and atypical respiratory bacteria (e.g. L. pneumophila, M. pneumoniae, C. pneumoniae) [57]. Notably, omadacycline retains activity against antibioticresistant Streptococcus spp. strains as well as potential activity against VRE, Acinetobacter spp., and ESBL producing organisms [57]. Omadacycline has also demonstrated promising activity against non-tuberculous mycobacterium such as Mycobacterium chelonae, Mycobacterium fortuitum, and Mycobacterium abscessus, prompting further studies into a potential future therapeutic niche $[57,58]$. 
Omadacycline has the tetracycline class warnings of tooth discoloration and inhibition of bone growth in children $<8$ years of age and CDAD [57]. The most common ADRs observed in clinical trials thus far have been nausea and vomiting [57].

This drug is currently approved for ABSSSI and CABP after demonstrating non-inferiority to linezolid and moxifloxacin, respectfully. The majority of these patients were initiated on therapy after being admitted to the hospital. Given its broad spectrum of activity, it may be useful in patients with high risk of MDR infection [57, 59-61]. Omadacycline is available in both IV and oral formulations, and as a once-daily, single agent effective in the treatment of ABSSSI and CABP, this drug may offer a favorable transitions-of-care option for these infections.

\section{Imipenem/cilistatin/relebactam}

Imipenem/cilistatin/relebactam (Recarbrio ${ }^{\mathrm{TM}}$ ) is a combination antibiotic incorporating both a broad-spectrum carbapenem and a novel beta-lactamase inhibitor approved in 2019 [62]. Imipenem/cilistatin is a well-known carbapenem antibiotic with a broad spectrum of activity and stand-alone stability against a number of beta-lactamases, including ESBL [62]. Cilistatin is formulated in combination with imipenem to prevent rapid degradation by dehydropeptidase-1, allowing for greater exposure [62]. Relebactam is a novel betalactamase inhibitor with a similar pharmacodynamic/pharmacokinetic profile to imipenem/cilistatin that can enhance the activity of imipenem/cilistatin against AmpC and KPCproducing Enterobacterales and $P$. aeruginosa [62]. Notably, imipenem/cilistatin/relebactam does not have reliable activity against metallo-beta-lactamases [12].

The side effect profile of imipenem/cilistatin/relebactam does not differ greatly from other beta-lactam antibiotics and has generally been associated with relatively mild nausea, vomiting, and diarrhea [62]. Similarly, this drug carries warnings for hypersensitivity reactions, CDAD, and seizures (especially if the patient is managed with valproic acid or divalproex) [62].

Imipenem/cilistatin/relebactam is currently indicated for the treatment of cUTI, cIAI, and HABP/VABP, indications largely carried over from the original imipenem/cilistatin [62]. However, imipenem/cilistatin/relebactam also demonstrated non-inferiority to piperacillin/tazobactam for HABP/ VABP with a separate trial on its own. [58, 63] Unlike other new gram-negative agents that initially sought approval for cUTIs, an early study observed this drug's efficacy and safety in cIAI, cUTI, and HABP/VABP in imipenem-resistant $P$. aeruginosa isolates compared to colistin + imipenem [64]. Thus, it has utility as a potential colistin-sparing treatment regimen for resistant $P$. aeruginosa. All patients in the trials were treated in acute care settings without mention of treatment in the emergency department.

\section{Cefiderocol}

Cefiderocol (Fetroja ${ }^{\mathrm{TM}}$ ) is a novel cephalosporin antibiotic approved in 2019. Like other cephalosporins, cefiderocol inhibits bacterial cell wall synthesis. However, it is uniquely classified as a siderophore antibiotic, as it utilizes ferric iron transport systems to take advantage of active bacterial uptake past the gram-negative outer lipopolysaccharide membrane, resulting in more drug exposure at the site of action [65].

Cefiderocol demonstrates broad coverage of gram-negative organisms, with a spectrum of activity that encompasses Acinetobacter baumannii, P. aeruginosa, Stenotrophomonas maltophilia, and resistant Enterobacterales, including all four Ambler class beta-lactamases (A, B, C, and D) [65]. In particular, the activity against Ambler class $\mathrm{B}$ metallo-betalactamases is a unique characteristic of this agent [65].

The adverse effect profile of cefiderocol does not differ greatly from other beta-lactam antibiotics and has generally been associated relatively mild nausea, vomiting, and diarrhea [65]. Similarly, this drug carries warnings for hypersensitivity reactions, CDAD, and seizures (especially if the patient is managed with valproic acid or divalproex) [65]. It is important to note that cefiderocol was found to have an increase in all-cause mortality in critically ill patients with multidrug-resistant gram-negative infections and subsequently carries that warning on its labeling [65].

Cefiderocol was first approved for cUTI after demonstrating non-inferiority to imipenem/cilistatin with no major problems observed [66]. The increase in all-cause mortality was noted in the CREDIBLE-CR study, in which cefiderocol was tested against best available therapy for MDR organisms responsible for nosocomial pneumonia, bloodstream infections, cUTI [67]. Although the cause of the increase in mortality is unknown, the greatest imbalance of death at the end of the study was seen in patients with A. baumannii infections or in patients with an APACHE II score of $\geq 16$ [67]. The increase in all-cause mortality currently limits the use of this agent for cUTI when other options are not available. Its use in bacteremia, sepsis, or nosocomial pneumonia cannot be formally recommended at this time.

\section{Conclusions}

Infections caused by MDR bacteria represent an ongoing threat to public health, and clinicians practicing in acute care settings will continue to play an important role in their treatment. The novel antibiotics reviewed here represent important additions to the antibiotic armamentarium and provide 
options for therapy where few otherwise exist. However, use of these drugs must be restricted to specific indications, as indiscriminate prescribing could accelerate resistance and degrade their future utility in treating MDR infections. Antimicrobial stewardship programs should be broadly implemented to increase clinician awareness of appropriate antibiotic use and local antibiotic resistance patterns. Institutional therapeutic guidelines and antibiograms can help clinicians choose the "right antibiotic, at the right dose, for the right duration, at the right time" [54]. Additionally, antibiotic therapy should be reviewed $48-72$ hours after initiation to assess for clinical response in concert with microbiological culture data when available, as opportunities may present to de-escalate or discontinue therapy. Within this context, it is important for acute care physicians to be familiar with novel and recently approved antibiotics so that they can better optimize their use in the treatment of drug-resistant infections.

\section{Funding None.}

\section{Declarations}

Conflict of interest The authors declare that they have no conflict of interest.

Ethical statement No patients were involved in the production of this manuscript.

Disclaimer The views expressed herein are those of the author(s) and do not reflect the official policy or position of Brooke Army Medical Center, the U.S. Army Medical Department, the U.S. Army Office of the Surgeon General, the Department of the Army, the Department of the Air Force, or the Department of Defense, or the U.S. Government.

The appearance of name-brand products in this article does not constitute endorsement by Brooke Army Medical Center, the U.S. Army Medical Department, the U.S. Army Office of the Surgeon General, the Department of the Army, the Department of the Air Force, or the Department of Defense, or the U.S. Government of the information, products, or services contained therein.

\section{References}

1. CDC (2019) Antibiotic resistance threats in the united states 2019. US Department of Health and Human Services. https:// doi.org/10.15620/cdc:82532

2. Nguyen L, Garcia J, Gruenberg K, MacDougall C (2018) Multidrug-resistant pseudomonas infections: Hard to treat, but hope on the horizon? Curr Infect Dis Rep 20:23. https://doi.org/10. 1007/s11908-018-0629-6

3. Pourmand A, Mazer-Amirshahi M, Jasani G, May L (2017) Emerging trends in antibiotic resistance: Implications for emergency medicine. Am J Emerg Med 35:1172-1176. https://doi. org/10.1016/j.ajem.2017.03.010

4. McGann P, Snesrud E, Maybank R, Corey B, Ong AC et al (2016) Escherichia coli harboring mcr-1 and blactx-m on a novel incf plasmid: First report of mcr-1 in the united states.
Antimicrob Agents Chemother 60:4420-4421. https://doi.org/ 10.1128/AAC.01103-16

5. Paterson DL, Bonomo RA (2005) Extended-spectrum beta-lactamases: a clinical update. Clin Microbiol Rev 18:657-686. https:// doi.org/10.1128/CMR.18.4.657-686.2005

6. Perletti G, Magri V, Cai T, Stamatiou K, Trinchieri A et al (2018) Resistance of uropathogens to antibacterial agents: emerging threats, trends and treatments. Arch Ital Urol Androl 90:85-96. https://doi.org/10.4081/aiua.2018.2.85

7. Zilahi G, Artigas A, Martin-Loeches I (2016) What's new in multidrug-resistant pathogens in the icu? Ann Intensive Care 6:96. https://doi.org/10.1186/s13613-016-0199-4

8. McConville TH, Sullivan SB, Gomez-Simmonds A, Whittier S, Uhlemann AC (2017) Carbapenem-resistant enterobacteriaceae colonization (cre) and subsequent risk of infection and 90-day mortality in critically ill patients, an observational study. PLoS ONE 12:e0186195. https://doi.org/10.1371/journal.pone.0186195

9. Magiorakos AP, Srinivasan A, Carey RB, Carmeli Y, Falagas ME et al (2012) Multidrug-resistant, extensively drug-resistant and pandrug-resistant bacteria: an international expert proposal for interim standard definitions for acquired resistance. Clin Microbiol Infect 18:268-281. https://doi.org/10.1111/j.1469-0691.2011. 03570.x

10 Tamma PDA, Samuel L et al (2020) Infectious diseases society of america antimicrobial resistant treatment guidance : Gramnegative bacterial infections. A focus on extended-spectrum $\beta$-lactamase producing enterobacterales (esbl-e), carbapenemresistant enterobacterales (cre), and pseudomonas aeruginosa with difficult-to-treat resistance (dtrp. Aeruginosa). Infectious Disease Society of America Practice Guidelines. Clin Infect Dis 72:169

11. Paterson DL, Bonomo RA (2005) Extended-spectrum $\beta$-lactamases: a clinical update. Clin Microbiol Rev 18:657-686. https://doi.org/10.1128/CMR.18.4.657-686.2005

12. Wong D, van Duin D (2017) Novel beta-lactamase inhibitors: unlocking their potential in therapy. Drugs 77:615

13. Sawa T, Kooguchi K, Moriyama K (2020) Molecular diversity of extended-spectrum $\beta$-lactamases and carbapenemases, and antimicrobial resistance. J Intensive Care 8:13-13. https://doi.org/10. 1186/s40560-020-0429-6

14. Cilloniz C, Dominedo C, Torres A (2019) Multidrug resistant gram-negative bacteria in community-acquired pneumonia. Crit Care 23:79. https://doi.org/10.1186/s13054-019-2371-3

15. Webb BJ, Sorensen J, Mecham I, Buckel W, Ooi L et al (2019) Antibiotic use and outcomes after implementation of the drug resistance in pneumonia score in ed patients with communityonset pneumonia. Chest 156:843-851. https://doi.org/10.1016/j. chest.2019.04.093

16. Dan S, Shah A, Justo JA, Bookstaver PB, Kohn J et al (2016) Prediction of fluoroquinolone resistance in gram-negative bacteria causing bloodstream infections. Antimicrob Agents Chemother 60:2265-2272. https://doi.org/10.1128/AAC.02728-15

17. Augustine MR, Testerman TL, Justo JA, Bookstaver PB, Kohn J et al (2017) Clinical risk score for prediction of extended-spectrum $\beta$-lactamase-producing enterobacteriaceae in bloodstream isolates. Infect Control Hosp Epidemiol 38:266-272. https://doi. org/10.1017/ice.2016.292

18. Shah A, Justo JA, Bookstaver PB, Kohn J, Albrecht H et al (2017) Application of fluoroquinolone resistance score in management of complicated urinary tract infections. Antimicrob Agents Chemother. https://doi.org/10.1128/AAC.02313-16

19. Kalil AC, Metersky ML, Klompas M, Muscedere J, Sweeney DA et al (2016) Management of adults with hospital-acquired and ventilator-associated pneumonia: 2016 clinical practice guidelines by the infectious diseases society of america and the american thoracic society. Clin Infect Dis 63:e61-e111. https://doi.org/10. 1093/cid/ciw353 
20. Doi Y, Park YS, Rivera JI, Adams-Haduch JM, Hingwe A et al (2013) Community-associated extended-spectrum beta-lactamaseproducing escherichia coli infection in the united states. Clin Infect Dis 56:641-648. https://doi.org/10.1093/cid/cis942

21. Frazee BW, Trivedi T, Montgomery M, Petrovic DF, Yamaji R et al (2018) Emergency department urinary tract infections caused by extended-spectrum beta-lactamase-producing enterobacteriaceae: many patients have no identifiable risk factor and discordant empiric therapy is common. Ann Emerg Med 72:449-456. https://doi.org/10.1016/j.annemergmed.2018.05.006

22. Talan DA, Takhar SS, Krishnadasan A, Abrahamian FM, Mower WR et al (2016) Fluoroquinolone-resistant and extended-spectrum beta-lactamase-producing escherichia coli infections in patients with pyelonephritis, united states(1). Emerg Infect Dis. https://doi. org/10.3201/eid2209.160148

23. Leone S, Damiani G, Pezone I, Kelly ME, Cascella M et al (2019) New antimicrobial options for the management of complicated intra-abdominal infections. Eur J Clin Microbiol Infect Dis 38:819-827. https://doi.org/10.1007/s10096-019-03533-y

24. Webb BJ, Dascomb K, Stenehjem E, Vikram HR, Agrwal N et al (2016) Derivation and multicenter validation of the drug resistance in pneumonia clinical prediction score. Antimicrob Agents Chemother 60:2652-2663. https://doi.org/10.1128/AAC. 03071-15

25. Ray S, Anand D, Purwar S, Samanta A, Upadhye KV et al (2018) Association of high mortality with extended-spectrum beta-lactamase (esbl) positive cultures in community acquired infections. $\mathbf{J}$ Crit Care 44:255-260. https://doi.org/10.1016/j.jcrc.2017.10.036

26. Giske CG, Monnet DL, Cars O, Carmeli Y, R. ReAct-Action on Antibiotic, (2008) Clinical and economic impact of common multidrug-resistant gram-negative bacilli. Antimicrob Agents Chemother 52:813-821. https://doi.org/10.1128/AAC.01169-07

27. Tumbarello M, Spanu T, Di Bidino R, Marchetti M, Ruggeri M et al (2010) Costs of bloodstream infections caused by escherichia coli and influence of extended-spectrum-beta-lactamase production and inadequate initial antibiotic therapy. Antimicrob Agents Chemother 54:4085-4091. https://doi.org/10.1128/AAC. 00143-10

28. Garnacho-Montero J, Gutierrez-Pizarraya A, Escoresca-Ortega A, Fernandez-Delgado E, Lopez-Sanchez JM (2015) Adequate antibiotic therapy prior to icu admission in patients with severe sepsis and septic shock reduces hospital mortality. Crit Care 19:302. https://doi.org/10.1186/s13054-015-1000-z

29. Zerbaxa [package insert]. 2014, Cubist Pharmaceuticals: Lexington, MA.

30. Wagenlehner FM, Umeh O, Steenbergen J, Yuan G, Darouiche RO (2015) Ceftolozane-tazobactam compared with levofloxacin in the treatment of complicated urinary-tract infections, including pyelonephritis: a randomised, double-blind, phase 3 trial (aspect-cuti). Lancet 385:1949-1956. https://doi.org/10.1016/S0140-6736(14) 62220-0

31. Solomkin J, Hershberger E, Miller B, Popejoy M, Friedland I et al (2015) Ceftolozane/tazobactam plus metronidazole for complicated intra-abdominal infections in an era of multidrug resistance: results from a randomized, double-blind, phase 3 trial (aspectciai). Clin Infect Dis 60:1462-1471. https://doi.org/10.1093/cid/ civ097

32. Kalil AC, Zavascki AP (2019) Can ceftolozane-tazobactam treat nosocomial pneumonia? Lancet Infect Dis 19:1266-1267. https:// doi.org/10.1016/S1473-3099(19)30523-7

33. Moya B, Zamorano L, Juan C, Ge Y, Oliver A (2010) Affinity of the new cephalosporin cxa-101 to penicillin-binding proteins of pseudomonas aeruginosa. Antimicrob Agents Chemother 54:3933-3937. https://doi.org/10.1128/AAC.00296-10

34 Gallagher JC, Satlin MJ, Elabor A, Saraiya N, McCreary EK et al (2018) Ceftolozane-tazobactam for the treatment of multidrug-resistant pseudomonas aeruginosa infections: a multicenter study. Open Forum Infect Dis 5:ofy280. https://doi.org/ 10.1093/ofid/ofy280

35. Sader HS, Flamm RK, Carvalhaes CG, Castanheira M (2020) Comparison of ceftazidime-avibactam and ceftolozane-tazobactam in vitro activities when tested against gram-negative bacteria isolated from patients hospitalized with pneumonia in united states medical centers (2017-2018). Diagn Microbiol Infect Dis 96:114833. https://doi.org/10.1016/j.diagmicrobio.2019.05.005

36. Hernandez-Tejedor A, Merino-Vega CD, Martin-Vivas A, Ruiz de Luna-Gonzalez R, Delgado-Iribarren A et al (2017) Successful treatment of multidrug-resistant pseudomonas aeruginosa breakthrough bacteremia with ceftolozane/tazobactam. Infection 45:115-117. https://doi.org/10.1007/s15010-016-0944-5

37. Avycaz [package insert]. 2019, GlaxoSmithKline Manufacturing: Verona, Italy.

38. Mazuski JE, Gasink LB, Armstrong J, Broadhurst H, Stone GG et al (2016) Efficacy and safety of ceftazidime-avibactam plus metronidazole versus meropenem in the treatment of complicated intra-abdominal infection: results from a randomized, controlled, double-blind, phase 3 program. Clin Infect Dis 62:1380-1389. https://doi.org/10.1093/cid/ciw133

39. Wagenlehner FM, Sobel JD, Newell P, Armstrong J, Huang X et al (2016) Ceftazidime-avibactam versus doripenem for the treatment of complicated urinary tract infections, including acute pyelonephritis: recapture, a phase 3 randomized trial program. Clin Infect Dis 63:754-762. https://doi.org/10.1093/cid/ciw378

40. Torres A, Rank D, Melnick D, Rekeda L, Chen X et al (2019) Randomized trial of ceftazidime-avibactam vs meropenem for treatment of hospital-acquired and ventilator-associated bacterial pneumonia (reprove): analyses per us fda-specified end points. Open Forum Infect Dis 6:ofz149. https://doi.org/10.1093/ofid/ ofz149

41. Falcone M, Paterson D (2016) Spotlight on ceftazidime/avibactam: a new option for mdr gram-negative infections. J Antimicrob Chemother 71:2713-2722. https://doi.org/10.1093/jac/dkw239

42. Buehrle DJ, Shields RK, Chen L, Hao B, Press EG et al (2016) Evaluation of the in vitro activity of ceftazidime-avibactam and ceftolozane-tazobactam against meropenem-resistant pseudomonas aeruginosa isolates. Antimicrob Agents Chemother 60:3227-3231. https://doi.org/10.1128/AAC.02969-15

43. Grupper M, Sutherland C, Nicolau DP (2017) Multicenter evaluation of ceftazidime-avibactam and ceftolozane-tazobactam inhibitory activity against meropenem-nonsusceptible pseudomonas aeruginosa from blood, respiratory tract, and wounds. Antimicrob Agents Chemother. https://doi.org/10.1128/AAC.00875-17

44. Vabomere [package insert]. 2020, Melinta Therapeutics, Inc.: Lincolnshire, IL.

45. Kaye KS, Bhowmick T, Metallidis S, Bleasdale SC, Sagan OS et al (2018) Effect of meropenem-vaborbactam vs piperacillintazobactam on clinical cure or improvement and microbial eradication in complicated urinary tract infection: the tango i randomized clinical trial. JAMA J Am Med Assoc 319:788-799. https://doi.org/10.1001/jama.2018.0438

46. Tsivkovski R, Lomovskaya O (2020) Biochemical activity of vaborbactam. Antimicrob Agents Chemother. https://doi.org/10. 1128/AAC.01935-19

47. Kaye KS, Bhowmick T, Metallidis S, Bleasdale SC, Sagan OS et al (2018) Effect of meropenem-vaborbactam vs piperacillintazobactam on clinical cure or improvement and microbial eradication in complicated urinary tract infection: the tango i randomized clinical trial. JAMA 319:788-799. https://doi.org/10. 1001/jama.2018.0438

48. Wunderink RG, Giamarellos-Bourboulis EJ, Rahav G, Mathers AJ, Bassetti M et al (2018) Effect and safety of meropenemvaborbactam versus best-available therapy in patients with 
carbapenem-resistant enterobacteriaceae infections: the tango ii randomized clinical trial. Infect Dis Ther 7:439-455. https://doi. org/10.1007/s40121-018-0214-1

49. Zemdri [package insert]. 2018, Achaogen, Inc.: South San Francisco, CA.

50. Shaeer KM, Zmarlicka MT, Chahine EB, Piccicacco N, Cho JC (2019) Plazomicin: a next-generation aminoglycoside. Pharmacotherapy 39:77-93. https://doi.org/10.1002/phar.2203

51. Wagenlehner FME, Cloutier DJ, Komirenko AS, Cebrik DS, Krause KM et al (2019) Once-daily plazomicin for complicated urinary tract infections. NEngl J Med 380:729-740. https://doi. org/10.1056/NEJMoa1801467

52. McKinnel J, Jubb A, Daikos GL (2019) Plazomicin for infections caused by carbapenem-resistant enterobacteriaceae. NEngl J Med 380:791-793

53. Xerava [package insert]. 2018, Tetraphase Pharmaceuticals, Inc.: Watertown, MA.

54 Zhanel GG, Cheung D, Adam H (2016) Review of eravacycline: a novel fluorocycline antibacterial agent. Drugs 76:567-588

55. Solomkin J, Evans D, Slepavicius A, Lee P, Marsh A et al (2017) Assessing the efficacy and safety of eravacycline vs ertapenem in complicated intra-abdominal infections in the investigating gramnegative infections treated with eravacycline (ignite 1) trial: a randomized clinical trial. JAMA Surg 152:224-232. https://doi.org/ 10.1001/jamasurg.2016.4237

56. Solomkin JS, Gardovskis J, Lawrence K, Montravers P, Sway A et al (2019) Ignite4: results of a phase 3, randomized, multicenter, prospective trial of eravacycline vs meropenem in the treatment of complicated intraabdominal infections. Clin Infect Dis 69:921929. https://doi.org/10.1093/cid/ciy1029

57. Nuzyra [package insert]. 2018, Paratek Pharmaceuticals, Inc.: Boston, MA.

58. Kaushik A, Ammerman NC, Martins O, Parrish NM, Nueremberger EL (2019) In vitro activity of new tetracycline analogs omadacycline and eravacycline against drug-resistant clinical isolates of mycobacterieum abscessus. Antimicrob Agents Chemother 63:e00470-e519. https://doi.org/10.1128/aac

59. Stets R, Popescu M, Gonong JR, Mitha I, Nseir W et al (2019) Omadacycline for community-acquired bacterial pneumonia. NEngl J Med 380:517-527. https://doi.org/10.1056/NEJMoa1800 201
60. O'Riordan W, Cardenas C, Shin E, Sirbu A, Garrity-Ryan L et al (2019) Once-daily oral omadacycline versus twice-daily oral linezolid for acute bacterial skin and skin structure infections (oasis2): a phase 3, double-blind, multicentre, randomised, controlled, non-inferiority trial. Lancet Infect Dis 19:1080-1090. https://doi. org/10.1016/S1473-3099(19)30275-0

61. O'Riordan W, Green S, Overcash JS, Puljiz I, Metallidis S et al (2019) Omadacycline for acute bacterial skin and skin-structure infections. NEngl J Med 380:528-538. https://doi.org/10.1056/ NEJMoa1800170

62. Recarbrio [package insert]. 2019, Merck \& Co., Inc.: Whitehouse Station, NJ.

63. Titov I, Wunderink RG, Roquilly A, Rodríguez Gonzalez D, David-Wang A et al (2020) A randomized, double-blind, multicenter trial comparing efficacy and safety of imipenem/cilastatin/ relebactam versus piperacillin/tazobactam in adults with hospitalacquired or ventilator-associated bacterial pneumonia (restore-imi 2 study). Clin Infect Dis. https://doi.org/10.1093/cid/ciaa803

64. Motsch J, Murta de Oliveira C, Stus V, Koksal I, Lyulko O et al (2020) Restore-imi 1: a multicenter, randomized, double-blind trial comparing efficacy and safety of imipenem/relebactam vs colistin plus imipenem in patients with imipenem-nonsusceptible bacterial infections. Clin Infect Dis 70:1799-1808. https://doi.org/ 10.1093/cid/ciz530

65. Fetroja [package insert]. 2019, Shionogi \& Co., Ltd: Osaka, Japan.

66. Portsmouth S, van Veenhuyzen D, Echols R, Machida M, Ferreira JCA et al (2018) Cefiderocol versus imipenem-cilastatin for the treatment of complicated urinary tract infections caused by gramnegative uropathogens: a phase 2 , randomised, double-blind, noninferiority trial. Lancet Infect Dis 18:1319-1328. https://doi.org/ 10.1016/s1473-3099(18)30554-1

67. Park F (2020) Study of s-649266 or best available therapy for the treatment of severe infections caused by carbapenem-resistant gram-negative pathogens- full text view, in ClincalTrials.gov. 2020.

Publisher's Note Springer Nature remains neutral with regard to jurisdictional claims in published maps and institutional affiliations. 